\title{
A Unique Case? Darier's Disease Presented as Porcupine-Like Appearance and the Observation on Acitretin Treatment*
}

\author{
Xi-Bao Zhang ${ }^{1 \#}$, Chang-Xing Li ${ }^{2}$, Xue-Mei Li ${ }^{1}$, Yu-Qing He ${ }^{1}$, Xiao Xu, ${ }^{1}$ Quan Luo ${ }^{1}$ \\ ${ }^{1}$ Department of Dermatology, Guangzhou Institute of Dermatology, Guangzhou, China; ${ }^{2}$ Department of Dermatology, Dongguan \\ Hospital of Chronic Disease, Dongguan, China. \\ Email: "lilichangxing@163.com
}

Received May $6^{\text {th }}, 2012$; revised June $10^{\text {th }}, 2012$; accepted June $29^{\text {th }}, 2012$

\begin{abstract}
Dyskeratosis follicularis (Darier's disease, DD) is rare autosomal dominant disease characterized by hyperkeratotic papules that coalesce into plaques and occur primarily in seborrheic or intertriginous areas. Associated findings include nail abnormalities. A 3-year-old boy presented with porcupine-like appearance for 2 years. The lesion from the back was taken for light microscopy and electron microscopy. He was treated with acitretin $(0.31 \mathrm{mg} / \mathrm{d}$ to $0.66 \mathrm{mg} / \mathrm{d}) \mathrm{for} 8$ years. Light microscopy and electron microscopy showed that the typical features of DD. The patient show good respond to the treatment. During 8 years treatment, the patient had dry mouth and pruritus. The skeletal abnormalities didn't happen in the patient. Evaluation of the serum lipid profile, liver function and renal function were within normal lever after treatment. Our findings showed that porcupine-like appearance is a unique pattern of DD. Acitretin may be a useful therapeutic agent in children with DD and less likely to cause skeletal problems.
\end{abstract}

Keywords: Darier's Disease; Acitretin

\section{Introduction}

The prevalence of DD has been estimated vary from one in 36,000 to one in 100,000 [1]. Clinically, the distinctive lesion is characterized by hyperkeratotic papules that coalesce into plaques and occur primarily in seborrheic, but also in intertriginous areas [1,2]. Coalescence of the papules produces irregular warty plaques or papillomatous masses, which, in the flexures, become hypertrophic, and malodorous with painful fissured. Associated abnormalities include nail abnormalities characterized by nail fragility, red and white longitudinal stripes and vshaped notches at the free margin of the nails [2]. In this article, we describe a case of DD with porcupine-like appearance. The patient showed good respond to the treatment of acitretin therapy.

\section{Case Report}

A 3-year-old boy had healthy skin at birth. At the age of 1 year, a brownish-red, keratotic papules and plaques appeared in his scalp, ears, perianal and genital areas. A biospy taken from his lesion revealed DD in the local

\footnotetext{
*Authors Xi-Bao Zhang and Chang-Xing Li contributed equally to this work.

"Corresponding author.
}

hospital. He showed no signifficant respond to the treatment of $0.025 \%$ retinoc acid cream or $0.1 \%$ mometasone furoate cream. The keratotic skin lesions worsened gradually as she grew. At 3 years of age, roughening, thickening and dryness of the keratotic skin lesions increased progressively to most parts of the body. Mentally the patient was normal and he had done well in school. His general health had been good. No other family members were affected.

On physical examination, the erythematous, keratotic, follicular papules were over his scalp, face and ear (Figure 1(a)). The disseminated heavy papillomatous hyperkeratosis was dark gray-brown and thickest on the legs, arms and trunk (Figures 1(b) and (c)). Nail involvement was subungual hyperkeratosis, dystrophy of the free margin of the nail and v-shaped notches at the free margin of the nails (Figure 1(d)).

\section{Materials and Methods}

Biopsy specimens were taken from affected skin on his back before treatment. The tissue specimens were studied under the light microscope as paraffin sections stained with hematoxylin-eosin or as $1 \mu \mathrm{m}$ epoxy resin-fixed sections. For electron microscopy, the tissue was fixed 2 


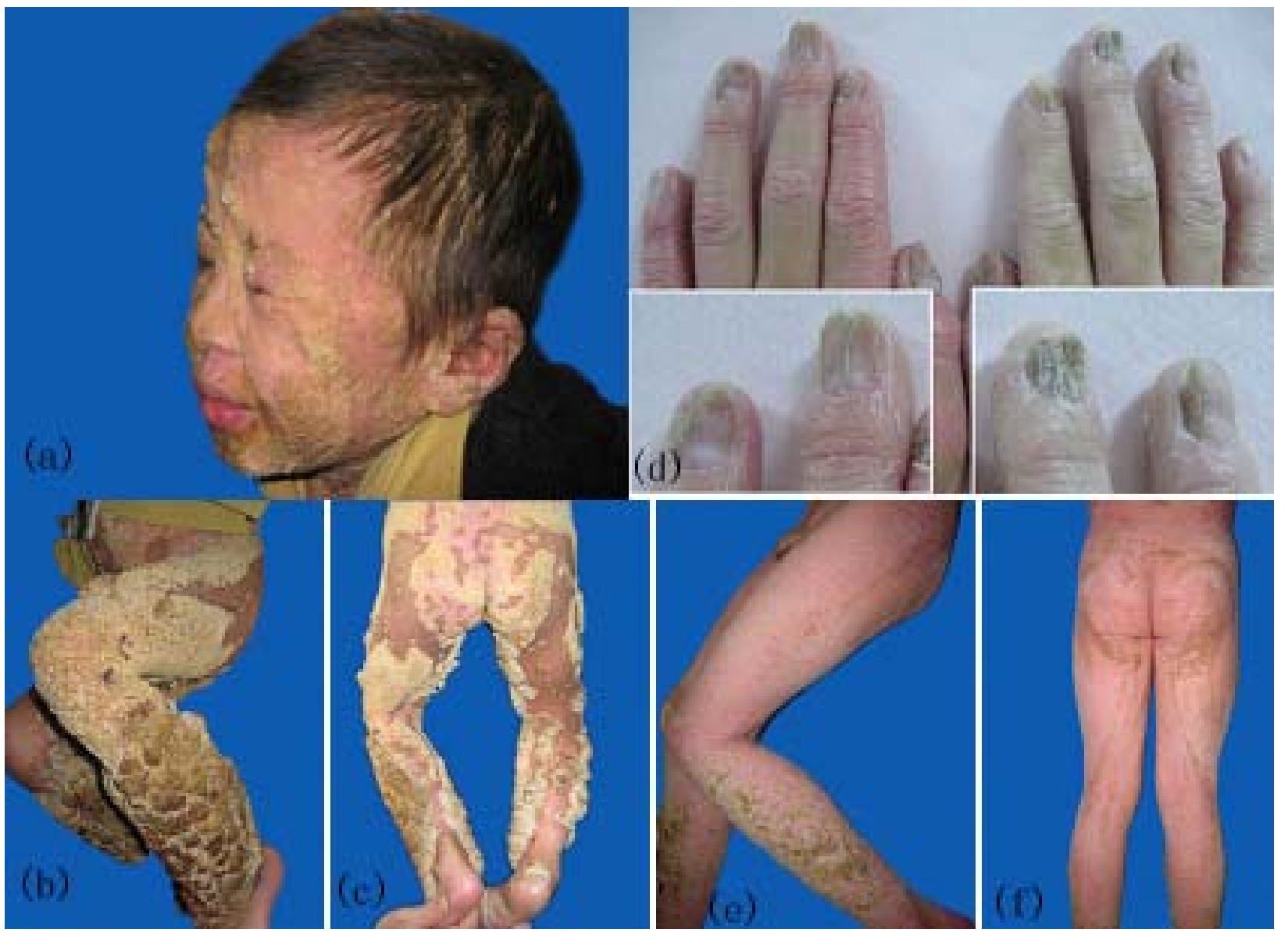

Figure 1. (a) The erythematous, keratotic, follicular papules were over his scalp, face and ear; (b) and (c) The disseminated heavy papillomatous hyperkeratosis was dark gray-brown and thickest on the legs, arms and trunk; (d) Nail involvement was subungual hyperkeratosis, dystrophy of the free margin of the nail and v-shaped notches at the free margin of the nails; (e) and (f) The patient's condition showed great improvement.

hours to 4 hours in 3\% glutaraldehyde and postfixed in $1 \%$ osmium tetroxide. The specimens were stained en bloc with uranyl acetate-lead citrate, dehydrated in a graded alcohol series, and embedded in epoxy resin (Epon812). Ultrathin sections were cut on an ultramicrotome, stained with uranyl acetate-lead citrate solutions, and examined with an electron microscope.

Assessment of efficacy:

The definitions of efficacy used in the present investigation were: 1) Cured in clinically. The improvement of skin lesions comes to over $90 \%$ been cured; the keratoderma is totally desquamated or light coarse remains on the skin. The patients can normally study and live in the society without any handicap; the life quality was improved completely; 2) Marked improvement. The improvement of skin lesions comes to $60 \%-90 \%$ and the patients can normally study and live, the life quality was improved remarkable; 3) Improvement. The improvement of skin lesions comes to $30 \%-60 \%$ and the patients can nearly study and live as normal the life quality was improved; 4) Failure. The skin improvement is less than $30 \%$ and the patients can't live in a normal life, the life quality was improved in a little.

Safety evaluation: Evaluate the safety according to the clinical side effects, changes in body weight and height, examination results of routine blood tests, liver and renal function, blood lipids and selective X-ray check for those cases been treated more than one year.

\section{Results}

Biopsies taken from back showed hyperkeratosis, parakeratosis, follicular plugging, irregular acanthosis, acantholytic keratinocytes forming corp ronds and corp grains (Figure 2), and an upward proliferation of papillae into the clefts. The direct immunofluorescence was negative.

Electron microscopic studies have demonstrated cell membrane damage, cytoplasmic vacuolization, dissolution of desmosome-keratin filament complexes and aggregation of keratin filaments around cell nucle. In the granular layer, the abnormality is cytoplasmic vacuolization. The affected cells display a spectrum of vacuoliza- 
tion ranging from a predominantly perinuclear distribution giving a foamy or cytolytic appearance. The tonofilaments and keratohyaline granules appear compressed into dense rings without attachments with neighboring cells (Figure 3(a)). Cells of the prickle layer, which are within lacunae, are separated from each other, having smooth rounded plasma membranes devoid of microvilli and desmosome attachments, and increasing aggregation of tonofilaments with perinuclear ditriution (Figures 3(b) - (d)).

Acitretin was used in the treatment of the case. The start treatment dose was $5 \mathrm{mg} /$ day $(0.38 \mathrm{mg} / \mathrm{kg} / \mathrm{d})$. The response was good and the patient's condition showed great improvement within 1 year. After 1 year, the dose of acitretin was increased to $10 \mathrm{mg} /$ day $(0.66 \mathrm{mg} / \mathrm{kg} / \mathrm{d})$, and the skin remained in good condition. After 2 years, the dose of acitretin was reduced to $6 \mathrm{mg} /$ day $(0.31 \mathrm{mg} /$ $\mathrm{kg} / \mathrm{d}$ ), and the skin remained in good condition within 2 years. After 4 years, the dose of acitretin was reduced to $5 \mathrm{mg} / \mathrm{day}(0.31 \mathrm{mg} / \mathrm{kg} / \mathrm{d})$, and the skin remained in good condition (Figures 1(e)-(f)) (Table 1).

During 8 years treatment, our patient had dry mouth and pruritus. The skeletal abnormalities didn't happen in the patient. Evaluation of the serum lipid profile, liver function and renal function were within normal lever after treatment.
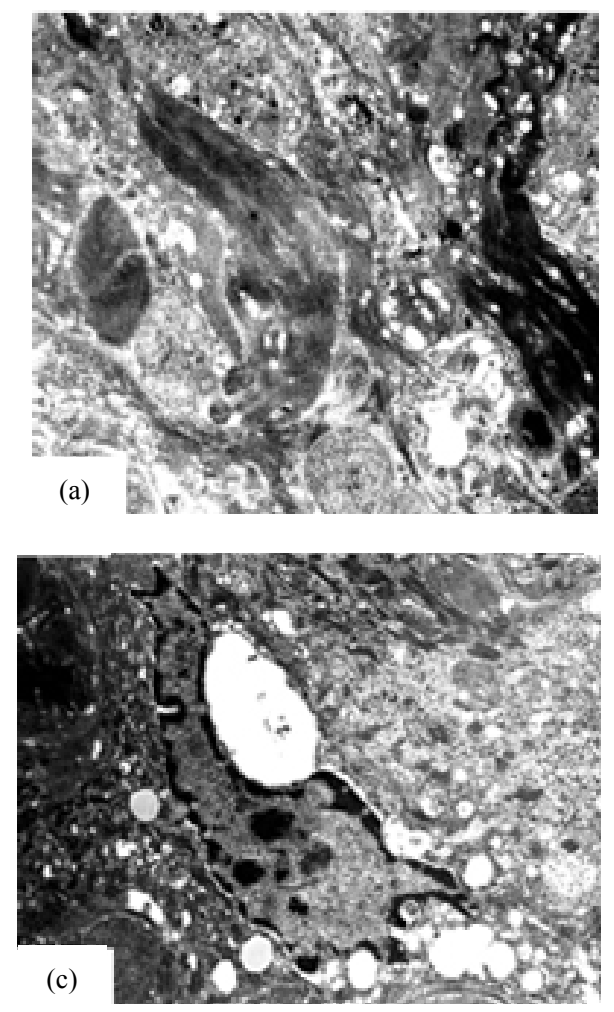

\section{Discussion}

The clinical expression of IH (Curth-Macklin) varies from palmoplantar keratoderma to a severe generalized involvement [3]. Histopathology shows granular cells with perinuclear oedema and shell formation, and binuclear cells, and the most prominent feature ultrastructurally are

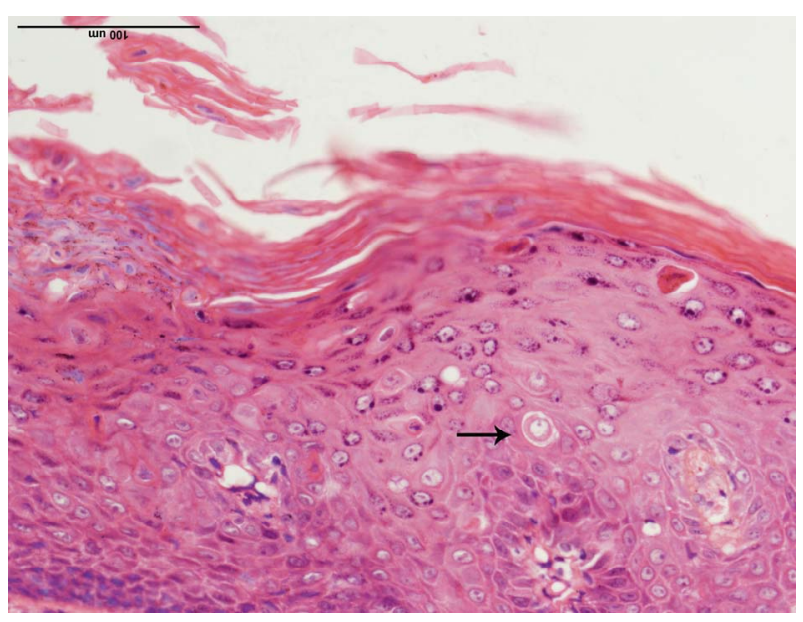

Figure 2. Histology showed hyperkeratosis, parakeratosis, follicular plugging, irregular acanthosis, acantholytic keratinocytes forming corp ronds and corp grains.
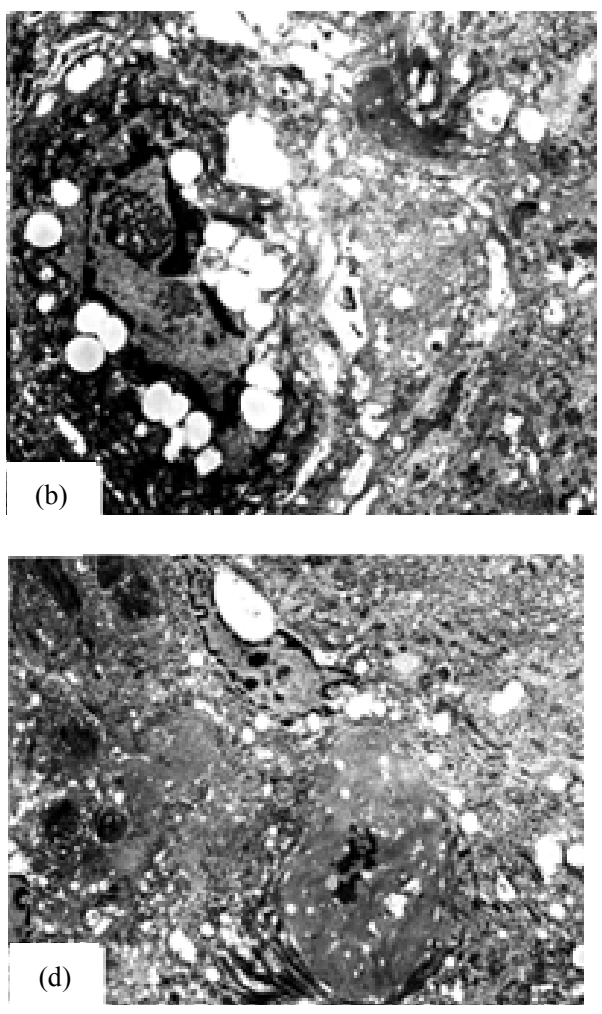

Figure 3. (a) The tonofilaments and keratohyaline granules appear compressed into dense rings without attachments with neighboring cells; (b-d) Cells of the prickle layer, which are within lacunae, are separated from each other, having smooth rounded plasma membranes devoid of microvilli and desmosome attachments, and increasing aggregation of tonofilaments with perinuclear ditriution. 
Table 1. Dose, improvement and side effects of acitritin.

\begin{tabular}{|c|c|c|c|c|c|c|c|c|c|}
\hline Age (y) & Height (cm) & Weight (kg) & Dose (mg/d) & improvement & skin & mucosa & bones & nerves & laboratory \\
\hline 3 & 90 & 13 & 5 & $\mathrm{C}$ & ++ & ++ & - & - & - \\
\hline 4 & 97 & 15 & 10 & $\mathrm{C}$ & ++ & ++ & - & - & - \\
\hline 5 & 103.5 & 17.5 & 10 & $\mathrm{C}$ & ++ & ++ & - & - & - \\
\hline 6 & 108 & 19 & 6 & B & ++ & ++ & - & - & - \\
\hline 7 & 114 & 22 & 6 & B & + & + & - & - & - \\
\hline 8 & 121.5 & 24.5 & 5 & B & + & + & - & - & - \\
\hline 9 & 126 & 26 & 5 & B & + & + & - & - & - \\
\hline 10 & 131 & 28 & 5 & A & + & + & - & - & - \\
\hline
\end{tabular}

+: Mild pruritus and dry; ++: Moderate pruritus and dry.

segregation of cytoplasmic components, perinuclear tonofilament shells and binuclear keratinocytes [3-5].

The clinical condition generalized epidermolytic hyperkeratosis, or bullous congenital ichthyosiform erythroderma, is characterized at birth and in the neonatal period by erythroderma, widespread bullae, and desquamation resulting in denuded skin. Focal hyperkeratosis may accompany these clinical findings. In infancy and into adulthood, the erythema and bullae are replaced with widespread hyperkeratosis, most prominent over joints, leading to a characteristic cobblestone appearance. Histologically, epidermolytic hyperkeratosis is characterized by vacuolization of the mid and upper spinous layer, large keratohyaline granules in vacuolated expanded granular cytoplasm of the keratinocytes containing an increased number of small and large irregularly shaped keratohyaline and trichohyaline-like bodies, and hyperkeratosis. The most prominent feature ultrastructurally in epidermolytic hyperkeratosis, is the suprabasal cells showed vacuolated cytoplasm and clumped keratin tonofilaments as in epidermolysis bullosa Dowling-Meara [6,7].

Our patient presented with disseminated dark graybrown and thickest heavy papillomatous hyperkeratosis on the legs, arms and trunk. The erythematous, keratotic, follicular papules were over his scalp, face and ear. Nail involvement was subungual hyperkeratosis, dystrophy of the free margin of the nail and v-shaped notches at the free margin of the nails. The clinical condition of our patient is resemblance to a porcupine and similar with IH (Curth-Macklin) and epidermolytic hyperkeratosis. It is difficult to distinguish these three disorders by clinical expression; however, the light microscopy and electron microscopic features are different. Histologic examination showed dyskeratosis with typical "corps ronds and grains," suprabasilar acanthosis, and hyperkeratosis, often with formation of papillae. The ultrastructural studies reveal breakdown of the desmosome-keratin filament complexes between keratinocytes. The light microscopy and electron microscopic features of our patient showed that the typical features of DD. Our patient was diagnosed as DD.

Despite much progress in understanding of the underlying abnormality in DD, disappointingly little has changed in our treatment armoury. Many patients with mild disease require no treatment other than simple emollient-like soap substitutes and moisturizers (in particular, those containing urea or lactic acid can reduce scaling and irritation) [8], with advice about the effects of sunshine. For those with more severe disease, oral retinoids (etretinate, acitretin, isotretinoin) are the most effective prescription. Most patients tolerate $0.6 \mathrm{mg} / \mathrm{kg}$ of acitrecin daily, but 10 $25 \mathrm{mg}$ daily is a reasonable starting dose and the dose can be increased gradually [8]. The clinical response is good in $90 \%$ of patients: Hyperkeratosis is reduced and papules are flattened, while malodour may also improve. However, retinoids are teratogenic and pregnancy must be avoided during and for a while after treatment. Retinoids treatment may cause skeletal abnormalities in children including osteoporosis, periosteal plucking, slender long bones and premature epiphyseal closure. A starting dose of acitretin of $0.38-0.66 \mathrm{mg} / \mathrm{kg} / \mathrm{d}$ was recommended and good responses were seen within $1-3$ years. The maintenance dose was titrated down to the 0.31 $\mathrm{mg} / \mathrm{kg} / \mathrm{d}$. During 8 years treatment, our patient had dry mouth and pruritus. The skeletal abnormalities didn't happen in the patient. Evaluation of the serum lipid profile, liver function and renal function were within normal lever after treatment.

In conclusion, the present study has demonstrated that porcupine-like appearance is a unique pattern of DD. Acitretin may be a useful therapeutic agent in children with DD and less likely to cause skeletal problems.

\section{REFERENCES}

[1] F. Loche, M. Carrière, H. P. Schwarze, B. Thédenat and J. 
Bazex, "Darier White Disease and Dermatofibrosarcoma Protuberans," Dermatology, Vol. 199, No. 3, 1999, p. 279. doi: $10.1159 / 000018268$

[2] S. Tavadia, E. Mortimer and C. S. Munro, "Genetic Epidemiology of Darier's Disease: A Population Study in the West of Scotland," British Journal of Dermatology, Vol. 146, No. 1, 2002, pp. 107-109. doi:10.1046/j.1365-2133.2002.04559.x

[3] H. O. Curth, F. H. J. Allen, U. W. Schnyder and I. Anton-Lamprecht, "Follow-Up of a Family Group Suffering from Ichthyosis Hystrix Type Curth-Macklin," Humangenetik, Vol. 17, No. 1, 1972, pp. 37-48. doi:10.1007/BF01789598

[4] L. Kanerva, J. Karvonen, A. Oikarinen, J. Lauharanta, A. Ruokonen and K. M. Niemi, "Ichthyosis Hystrix (CurthMacklin). Light and Electron Microscopic Studies Performed before and after Etretinate Treatment," Archives of Dermatology, Vol. 120, No. 9, 1984, pp. 1218-1223. doi:10.1001/archderm.1984.01650450100030

[5] K. M. Niemi, I. Virtanen, L. Kanerva and M. Muttilainen, "Altered Keratin Expression in Ichthyosis Hystrix CurthMacklin. A Light and Electron Microscopic Study," Archives of Dermatological Research, Vol. 282, No. 4, 1990, pp. 227-233. doi:10.1007/BF00371641

[6] A. L. Ingrun, "Ultrastructural Identification of Basic Abnormalities as Clues to Genetic Disorders of the Epidermis," Journal of Investigative Dermatology, Vol. 103, No. 5, 1994, pp. 6-12. doi:10.1111/1523-1747.ep12398887

[7] A. B. Ackerman and G. Goldman, "Combined Epidermolytic Hyperkeratosis and Focal Acantholytic Dyskeratosis," Archives of Dermatology, Vol. 109, No. 3, 1974, pp. 385-386. doi:10.1001/archderm.1974.01630030043010

[8] S. Bruge, "Management of Darier's Disease," Clinical and Experimental Dermatology, Vol. 24, No. 2, 1999, pp. 53-56. doi:10.1046/j.1365-2230.1999.00416.x 